\title{
Department of Physics
}

\section{Papers from the Department of Physics}

Year 2004

\section{Halo neutrons and the beta decay of ${ }^{11} \mathrm{Li}$}

\author{
F. Sarazin \\ J. S. Al-Khalili \\ G. C. Ball \\ G. Hackman \\ P. M. Walker, University of Surrey \\ R. A. E. Austin \\ B. Eshpeter \\ P. Finlay \\ P. E. Garrett \\ G. F. Grinyer \\ K. A. Koopmans \\ W. D. Kulp \\ J. R. Leslie \\ D. Melconian \\ C. J. Osborne \\ M. A. Schumaker \\ H. C. Scraggs \\ J. Schwarzenberg \\ M. B. Smith \\ C. E. Svensson \\ J. C. Waddington \\ J. L. Wood
}

This paper is posted at Surrey Scholarship Online.

http://epubs.surrey.ac.uk/physicspapers/43 


\title{
Halo neutrons and the $\beta$ decay of ${ }^{11} \mathrm{Li}$
}

\author{
F. Sarazin, ${ }^{1,2, *}$ J. S. Al-Khalili, ${ }^{2,3}$ G. C. Ball, ${ }^{2}$ G. Hackman, ${ }^{2}$ P. M. Walker, ${ }^{2,3}$ R. A. E. Austin, ${ }^{4, \dagger}$ B. Eshpeter, ${ }^{2}$ P. Finlay, ${ }^{5}$ \\ P. E. Garrett, ${ }^{6}$ G. F. Grinyer ${ }^{5}$ K. A. Koopmans, ${ }^{4}$ W. D. Kulp,${ }^{7}$ J. R. Leslie, ${ }^{8}$ D. Melconian, ${ }^{9}$ C. J. Osborne, ${ }^{2, \$}$ \\ M. A. Schumaker, ${ }^{5}$ H. C. Scraggs, ${ }^{2}{ }^{8}$ J. Schwarzenberg, ${ }^{10}$ M. B. Smith, ${ }^{2}$ C. E. Svensson, ${ }^{5}$ J. C. Waddington, ${ }^{4}$ and J. L. Wood ${ }^{7}$ \\ ${ }^{1}$ Department of Physics, Colorado School of Mines, Golden, Colorado 80401, USA \\ ${ }^{2}$ TRIUMF, 4004 Wesbrook Mall, Vancouver, British Columbia, Canada V6T 2A3 \\ ${ }^{3}$ Department of Physics, University of Surrey, Guildford, Surrey GU2 7XH, United Kingdom \\ ${ }^{4}$ Department of Physics and Astronomy, McMaster University, Hamilton, Ontario, Canada L8S $4 K 1$ \\ ${ }^{5}$ Department of Physics, University of Guelph, Guelph, Ontario, Canada N1G 2 W1 \\ ${ }^{6}$ Lawrence Livermore National Laboratory, Livermore, California 94551, USA \\ ${ }^{7}$ School of Physics, Georgia Institute of Technology, Atlanta, Georgia 30332, USA \\ ${ }^{8}$ Department of Physics, Queen's University, Kingston, Ontario, Canada K7L 3N6 \\ ${ }^{9}$ Department of Physics, Simon Fraser University, Burnaby, British Columbia, Canada V5A 1 S6 \\ ${ }^{10}$ Department of Nuclear Physics, University of Vienna, Waehringerstrasse 17, Vienna, 1090 Austria
}

(Received 12 July 2004; published 21 September 2004)

\begin{abstract}
The $\beta$ decay of ${ }^{11} \mathrm{Li}$ has been investigated at TRIUMF-ISAC using a high-efficiency array of Compton suppressed HPGe detectors. From a line-shape analysis of the Doppler-broadened peaks observed in the ${ }^{10} \mathrm{Be}$ $\gamma$ spectrum, both the half-lives of states in ${ }^{10} \mathrm{Be}$ and the energies of the $\beta$-delayed neutrons feeding those states were obtained. Furthermore, it was possible to determine the excitation energies of the parent states in ${ }^{11} \mathrm{Be}$ with uncertainties comparable to those obtained from neutron spectroscopy experiments. These data suggest that the $\beta$ decay to the $8.81 \mathrm{MeV}$ state in ${ }^{11} \mathrm{Be}$ occurs in the ${ }^{9} \mathrm{Li}$ core and that one neutron comprising the halo of ${ }^{11} \mathrm{Li}$ survives in a halolike configuration after the $\beta$-delayed neutron emission from this level.
\end{abstract}

DOI: 10.1103/PhysRevC.70.031302

PACS number(s): 23.20.- g, 23.40.-s, 27.20.+n

The concept of loosely bound quantum particles having large spatial extent, beyond classical limits, is key to understanding a variety of physical systems [1]. The neutron halo of ${ }^{11} \mathrm{Li}$ has been the subject of intense studies since it was first proposed [2] to explain the abnormally large ratio of the matter-to-charge radius of ${ }^{11} \mathrm{Li}$ [3]. In particular, the study of the $\beta$ decay of ${ }^{11} \mathrm{Li}$ is expected to shed light on how the weak interaction affects the two neutrons composing the halo. Presently [4], it is believed that the two valence neutrons are effectively decoupled from the core nucleus $\left({ }^{9} \mathrm{Li}\right)$. As a result, if the $\beta$ decay occurs in the core, the neutrons of the halo could in principle remain unaffected, but would then "react" to the fact that the core has changed [5]. In this paper, we discuss the striking possibility that one neutron of the ${ }^{11} \mathrm{Li}$ halo could survive in a halo configuration through the full $\beta$-delayed one-neutron emission process.

While the large ${ }^{11} \mathrm{Li}_{-}{ }^{11} \mathrm{Be}$ mass difference $\left(Q_{\beta}\right.$ $=20.61 \mathrm{MeV})$ and the low neutron separation energy in

${ }^{11} \mathrm{Be}\left(S_{n}=0.503 \mathrm{MeV}\right)$ open a large number of decay channels in the $\beta$ decay of ${ }^{11} \mathrm{Li}$ (see [6] and references therein), most of the $\beta$-decay strength leads to classical channels, namely by $\beta$ emission to the only bound excited state in ${ }^{11} \mathrm{Be}$

\footnotetext{
*Corresponding author. Electronic address: fsarazin@mines.edu

†Present address: Department of Physics and Astronomy, Saint Mary's University, Halifax NS, Canada B3H 3C3.

"Present address: Max Planck Institut für Kernphysik, Heidelberg, 69117 Germany.

${ }^{\S}$ Present address: Department of Physics, University of Liverpool, L69 Liverpool, 7ZE United Kingdom.
}

at $320 \mathrm{keV}(6.3 \%)$ and, by $\beta$-delayed one-neutron emission $(\beta n)(87.6 \%)$, to bound excited states in ${ }^{10} \mathrm{Be}[7]$. The latter decay path has been investigated recently by different experiments using $\beta-\gamma$ and $\beta-n-\gamma$ coincidences [6-9]. Discrepancies exist among these experiments. The $\gamma$-ray intensities of the transitions in ${ }^{10} \mathrm{Be}$ are found to be significantly different, while some ambiguities arise from the interpretation of the neutron spectra [6].

To study the $\beta$-delayed neutron emission of neutron-rich nuclei, it is often necessary to detect both the neutron(s) and $\gamma$ rays emitted in order to determine the excitation energies of the levels populated in progeny nuclei. However, in the present case, the level structure of ${ }^{10} \mathrm{Be}$ is well known up to the one-neutron separation energy at $6.812 \mathrm{MeV}[10,11]$, and the $\gamma$ peaks resulting from the decay of the ${ }^{10} \mathrm{Be}$ excited states have characteristic Doppler-broadened line shapes due to the ${ }^{10} \mathrm{Be}$ recoil induced by the $\beta$-delayed neutron emission. It is therefore possible, in principle, to make a complete analysis of all the ${ }^{11} \mathrm{Li} \beta n$ branches that feed the excited states of ${ }^{10} \mathrm{Be}$ through an analysis of the $\gamma$-ray energy spectra. In this way, the neutron energy spectrum may be determined, complementing and adding to the information obtained by direct neutron observation. An attempt to analyze these line shapes was made by Fynbo et al. [12] using the $\gamma$ spectrum obtained by Borge et al. [7] and again, more recently, with a set of data obtained at ISOLDE [9].

The $\beta$ decay of ${ }^{11} \mathrm{Li}$ was investigated at TRIUMF-ISAC [13] with the $8 \pi$ spectrometer, an array of 20 Comptonsuppressed high-purity germanium (HPGe) detectors [14]. ${ }^{11} \mathrm{Li}$ was produced by bombarding a $22 \mathrm{~g} / \mathrm{cm}^{2}$ Ta target with a $500 \mathrm{MeV}$ proton beam. A pure $30.4 \mathrm{keV}{ }^{11} \mathrm{Li}$ beam of 


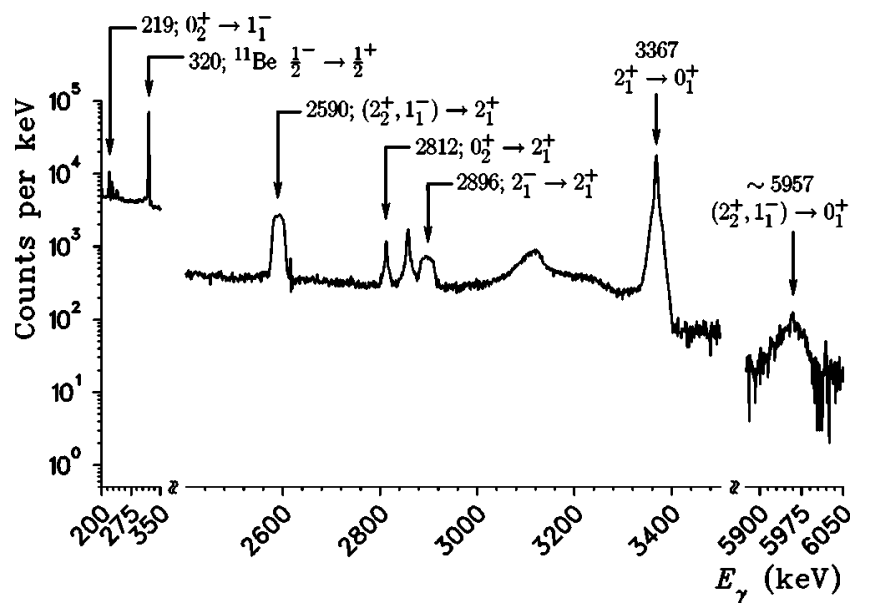

FIG. 1. Compton-suppressed $\gamma$ spectrum following the $\beta$ decay of ${ }^{11} \mathrm{Li}$. Only the relevant parts that contain the $\gamma$ transitions observed in ${ }^{10} \mathrm{Be}$ and in ${ }^{11} \mathrm{Be}$ are shown (room background subtracted).

about a thousand ions per second was extracted by surface ionization from the target and implanted into a 0.2 -mm-thick $\mathrm{Al}$ foil at the centre of the $\gamma$ detector array. A total of $8.1 \mathrm{M} \gamma$ singles were collected over a 2.5 day period.

The $\gamma$ peaks corresponding to the $\beta$ and $\beta n$ decay of ${ }^{11} \mathrm{Li}$ are shown in Fig. 1. Besides the known $320 \mathrm{keV}$ transition to the ground state of ${ }^{11} \mathrm{Be}$, the other $\gamma$ rays labeled on the figure are identified as known transitions in ${ }^{10} \mathrm{Be}$.

The relative efficiency of the array up to $3.4 \mathrm{MeV}$ was measured to an uncertainty of $\pm 2 \%$ with calibrated sources of ${ }^{152} \mathrm{Eu}$ and ${ }^{56} \mathrm{Co}$ fit using the RadWare package [15]. The relative efficiency at high energies (up to about $8 \mathrm{MeV}$ ) was obtained from known transitions in the $\beta$ decay of ${ }^{11} \mathrm{Be}$ [16]. A GEANT [17] simulation was also developed to predict the relative efficiency of the array at high energies. This simulation agreed with experiment to about $10 \%$ at $6 \mathrm{MeV}$. The $\gamma$-ray energies and relative intensities observed in the present experiment are summarized in Table I.

A new $\gamma$ ray was observed at $2896.0(5) \mathrm{keV}$ corresponding to the decay of the $6264 \mathrm{keV}, 2^{-}$level in ${ }^{10} \mathrm{Be}$ to the $2_{1}^{+}$ level at $3368 \mathrm{keV}$. In addition, an unresolved doublet was seen at $\sim 5957 \mathrm{keV}$ corresponding to the ground-state decay of the known $\left(2_{2}^{+}, 1^{-}\right)$doublet in ${ }^{10} \mathrm{Be}$. Both of these transitions were also reported recently by Fynbo et al. [9]. The relative intensities observed in the present experiment are a factor 2-5 times more precise than all previous data [6-9], sufficient to resolve previous discrepancies. In general, the results are in agreement with those obtained by Borge et al. [7] and Aoi et al. [8]. However, large discrepancies (about a factor of 2) remain for the 2590 and $2812 \mathrm{keV}$ transitions reported by Morrissey et al. [6] and the 219 and $5957 \mathrm{keV}$ $\gamma$-ray peaks seen by Fynbo et al. [9].

The excitation energies of the $1^{-}, \mathrm{O}_{2}^{+}$, and $2^{-}$states in ${ }^{10} \mathrm{Be}$ were determined by fitting the Doppler broadened line shapes and correcting for the recoil effect. The energies of both the $1^{-}$and $0_{2}^{+}$states were found to be about $1 \mathrm{keV}$ above the previous values $[10,11]$, whereas the precision of the energy of the $2^{-}$state was improved by a factor of 10 (see Table I).
TABLE I. Excited states in ${ }^{10} \mathrm{Be}$ and in ${ }^{11} \mathrm{Be}$. Energy $\left(E_{\gamma}\right)$, intensity, and branching ratio (BR) of the $\gamma$ transitions in ${ }^{10} \mathrm{Be}$ observed after the $\beta$-delayed neutron emission of ${ }^{11} \mathrm{Li}$. The intensities are normalized to the $3367 \mathrm{keV}$ transition.

\begin{tabular}{lcccc}
\hline \hline$E_{\text {Level }}(\mathrm{keV})$ & $E_{\gamma}(\mathrm{keV})$ & Assignment & Intensity & BR (\%) \\
\hline $320.04(1)$ & $320.0(5)$ & ${ }^{11}{\mathrm{Be}: \frac{1}{2}^{-} \rightarrow \frac{1}{2}^{+}}$ & $23.3(12)$ & \\
$3368.03(3)$ & $3367.1(5)$ & $2_{1}^{+} \rightarrow 0_{1}^{+}$ & $100(2)$ & \\
$5958.39(5)$ & $2590.3(5)$ & $2_{2}^{+} \rightarrow 2_{1}^{+}$ & $26.5(6)^{\mathrm{a}}$ & $90(3)^{\mathrm{a}}$ \\
& $5956.5(10)$ & $2_{2}^{+} \rightarrow 0_{1}^{+}$ & $2.9(9)^{\mathrm{a}}$ & $10(3)^{\mathrm{a}}$ \\
$5961.1(5)^{\mathrm{b}}$ & $2592.7(5)^{\mathrm{c}}$ & $1^{-} \rightarrow 2_{1}^{+}$ & $0.7(3)^{\mathrm{a}}$ & $35(15)^{\mathrm{a}}$ \\
& $5959.2(10)^{\mathrm{c}}$ & $1^{-} \rightarrow 0_{1}^{+}$ & $1.3(3)^{\mathrm{a}}$ & $65(15)^{\mathrm{a}}$ \\
$6180.3(5)^{\mathrm{b}}$ & $219.2(5)$ & $0_{2}^{+} \rightarrow 1_{1}^{-}$ & $2.04(11)$ & $39(2)$ \\
& $2811.8(5)$ & $0_{2}^{+} \rightarrow 2_{1}^{+}$ & $3.13(10)$ & $61(2)$ \\
$6264.5(5)^{\mathrm{b}}$ & $2896.0(5)$ & $2^{-} \rightarrow 2_{1}^{+}$ & $6.21(16)$ & 100 \\
\hline \hline
\end{tabular}

$\overline{\bar{a} \text { Intensities/branching ratios determined from fitting doublets (see }}$ text).

${ }^{b}$ Level energies determined in this experiment (see text).

${ }^{c}$ Energies determined from fitting doublets (see text).

A Monte Carlo simulation has been developed to analyze the complex shape of the $\gamma$ lines observed in this experiment. The line shape of a given $\gamma$ peak mainly depends on the energies and relative intensities of all the neutron branches feeding, directly or indirectly, the state from which the $\gamma$ transition arises, and on the lifetimes of the excited state populated in ${ }^{10} \mathrm{Be}$. The line shape of the peaks can also be affected by the angular correlation between the recoil (neutron) and the $\gamma$ ray [12]. Using the energy loss data computed in the latest version of SRIM [18], the implantation profile of ${ }^{11} \mathrm{Li}$ atoms in the $\mathrm{Al}$ foil was generated and the $\beta$-delayed one-neutron emission simulated. The recoil was assumed to be emitted isotropically in the laboratory frame. The treatment of the recoil escaping the foil, before it stops, in the backward angles is included in the simulation, as it affects the line shape of the peaks. However, in all cases, the recoil travels only a few micrometers before the $\gamma$ rays are emitted and therefore the efficiency of the HPGe detector array is not affected. The intrinsic response of the HPGe detectors was approximated with a Gaussian shape, with an energy-dependent width $\sigma$. No significant asymmetry was found in the data when comparing the $\gamma$ spectra of the individual HPGe detectors. The spectra from all 20 detectors were summed and the analysis was performed on the resulting spectrum. To compare the data with the Monte Carlo simulation, the Compton background underneath the peaks was subtracted with the appropriate error propagation. The neutron feeding intensities deduced from the line-shape analysis, together with the $\gamma$-ray intensities, are shown in Fig. 2.

No angular correlation between the recoil and the $\gamma$ ray is expected in the line shapes of the 2812 and $2896 \mathrm{keV}$ transitions corresponding to the decay of the $6180 \mathrm{keV}, 0_{2}^{+}$and $6264 \mathrm{keV}, 2^{-}$states, respectively, to the $3368 \mathrm{keV}, 2_{1}^{+}$level. This arises from the fact that the $2^{-}$state is believed to be fed mainly by the emission of an $\ell=0$ neutron from a $(3 / 2,5 / 2)^{-}$state in ${ }^{11} \mathrm{Be}$ (discussed later in the paper), 


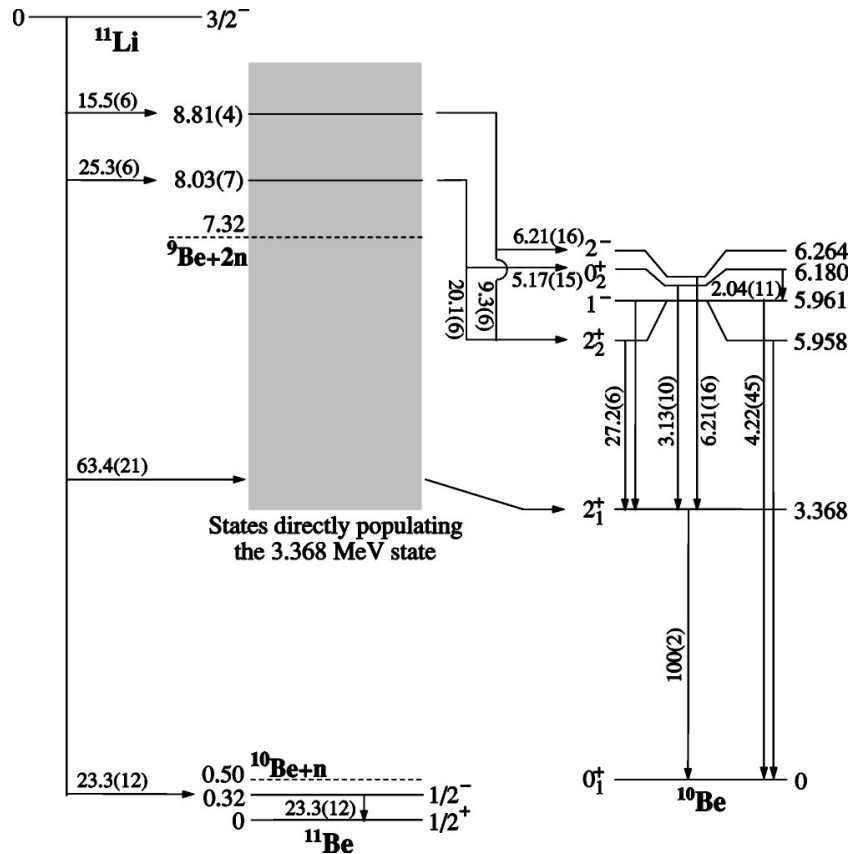

FIG. 2. Decay scheme of ${ }^{11} \mathrm{Li}$ deduced from this work. The energies of the first two excited states in ${ }^{10} \mathrm{Be}$ were taken from [11]. All transitions are labeled with $\gamma$-ray intensities, normalized to the $3367 \mathrm{keV}$ transition (100). The intensity breakdown of the decay of the $\left(1^{-}, 2_{2}^{+}\right)$doublet can be found in Table I.

whereas the $\gamma$-ray emission from the $0_{2}^{+}$state is naturally isotropic. The remaining free parameters are the energy $E_{\gamma}$ of the transition, the half-life $T_{1 / 2}$ of its state of origin, and the energy of the neutron that gives rise to the observed Doppler broadening (or the excitation energy $E_{X}$ of the parent state in $\left.{ }^{11} \mathrm{Be}\right)$.
The line shapes of both peaks were produced in the Monte Carlo simulation as a function of $E_{\gamma}, T_{1 / 2}$, and $E_{X}$, and compared to the data using a $\chi^{2}$ minimization method. The procedure was iterated until the minimum was found for the best triplet $\left(E_{\gamma}, E_{X}, T_{1 / 2}\right)$ that fits the data; acceptable fits (reduced $\left.\chi^{2} / \nu \sim 1\right)$ were obtained for both transitions. For the $6180 \mathrm{keV}, 0_{2}^{+}$state, the half-life was found to be $T_{1 / 2}$ $=870 \pm 70$ (stat) \pm 160 (syst) fs, consistent with the previous published value of $800_{-200}^{+300}$ fs $[10,11]$, for a neutron originating from an 8.03(7) $\mathrm{MeV}$ state in ${ }^{11} \mathrm{Be}$. Similarly, for the $6264 \mathrm{keV}, 2^{-}$state, the half-life was found to be $T_{1 / 2}$ $=85 \pm 6$ (stat) \pm 10 (syst) fs for a neutron originating from an $8.81(4) \mathrm{MeV}$ state in ${ }^{11} \mathrm{Be}$. The systematic error on the halflives was estimated by considering a $\pm 10 \%$ deviation from the SRIM tabulated energy loss of ${ }^{10} \mathrm{Be}$ in aluminum. The best fits for the 2812 and $2896 \mathrm{keV}$ transitions are shown in Fig. 3. No need was found to add any other neutron branch to fit the experimental line shape of either transition. This analysis unambiguously confirms the existence of the $8.03 \mathrm{MeV}$ state suggested by Aoi [8] (but not confirmed by Morrissey [6]). Furthermore, the uncertainties in the excitation energies of the parent states in ${ }^{11} \mathrm{Be}$ obtained in this experiment are, remarkably, comparable to those obtained from neutron spectroscopy experiments.

The analysis of the transitions originating from the $\left(1^{-}, 2_{2}^{+}\right)$doublet of states is more complex, because it involves the indirect feeding of the $1^{-}$state from the $0_{2}^{+}$state as well as the direct feeding of one or both states of the doublet. We note that the branching ratios from the $2_{2}^{+}$and $1^{-}$states to the $2_{1}^{+}$state were previously found to be $>90 \%$ and $17_{-10}^{+6} \%$ respectively $[10,11]$. The relative intensities of the 2590 and $5957 \mathrm{keV}$ transitions observed in the present experiment (see Fig. 2) strongly suggest that most, if not all, of the direct feeding proceeds through the $2_{2}^{+}$member of the doublet. We
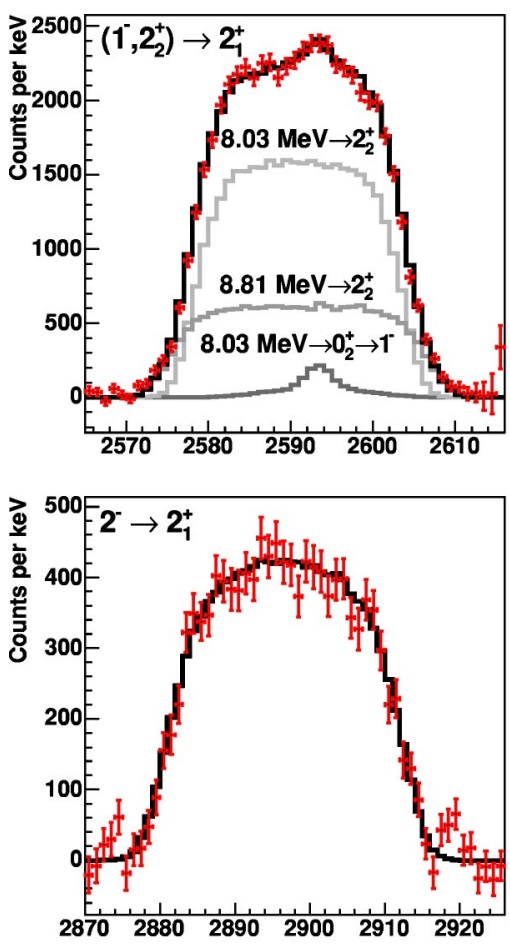
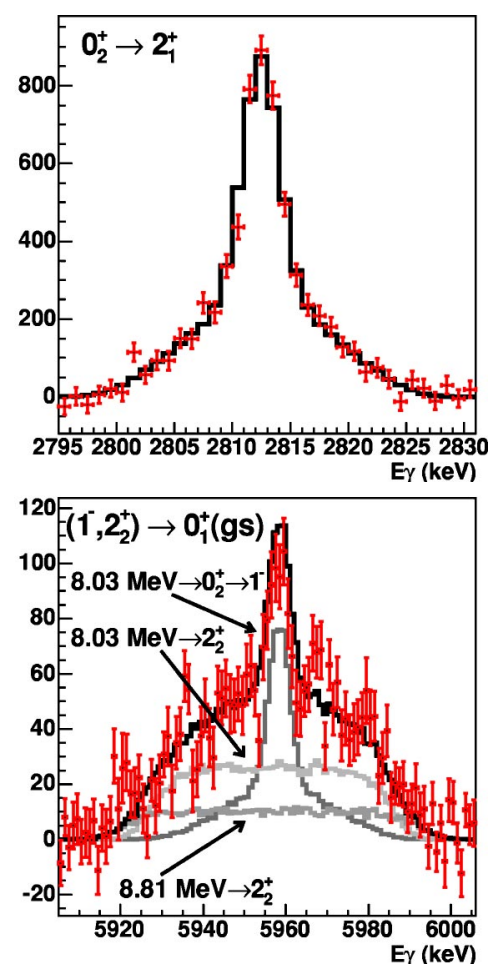

FIG. 3. (Color online) Comparison between the experimental data and the best-fit obtained by the Monte Carlo simulation (black line). For the transitions involving the $\left(1^{-}, 2_{2}^{+}\right)$doublet, the three contributions discussed in the text are also shown. 
did not find any compelling evidence that the $1^{-}$state is directly populated. For example, adding a direct feeding component to the $1^{-}$state provokes a shift in the center of gravity of the simulated $2590 \mathrm{keV}$ transition line shape, resulting in a larger $\chi^{2}$. The analysis is, however, limited by the relatively poor quality of the $5957 \mathrm{keV}$ transition line shape, which would be the most sensitive to a $1^{-}$direct branch. We disagree with the analysis of [9], which assumes that a direct feeding of the $1^{-}$state is responsible for the broad component of the $5957 \mathrm{keV}$ transition. In our case, this broad component is attributed to the direct feeding of the $2_{2}^{+}$state. In principle, the half-life of the $1^{-}$state could be deduced from the analysis of the sharp component of the $5957 \mathrm{keV}$ transition. However, this line shape is dominated by the effect of the half-life of the $0_{2}^{+}$state. We can only presume that the half-life of the $1^{-}$state is smaller than a few hundred femtoseconds.

The parity change necessary to connect the states in ${ }^{11} \mathrm{Be}$, fed by the $\beta$ decay of ${ }^{11} \mathrm{Li}$, to the $2_{2}^{+}$state in ${ }^{10} \mathrm{Be}$ implies the emission of an odd- $\ell$ neutron, most likely $\ell=1$. It is therefore necessary to consider angular correlation effects. Careful analysis of these effects shows a strong correlation of the half-life with the $A_{2}$ parameter, as defined in [19], but no significant correlation with the other free parameters of the simulation: $E_{\gamma}, E_{X}$ and, in this case, the branching ratios. Therefore, the simulations were first performed assuming no angular correlation $\left(A_{2}=0\right)$. It was found that the neutron branch from the $8.03 \mathrm{MeV}$ state suggested by [8] cannot account for all the Doppler broadening of the $2590 \mathrm{keV}$ peak, resulting in a poor $\chi^{2} / \nu \sim 8$. The situation is greatly improved $\left(\chi^{2} / \nu \sim 1\right)$ by adding a neutron branch from the 8.81 MeV state with an appropriate intensity. The excitation energy of the $1^{-}$state was deduced from the centroid of the indirect contribution of the $0_{2}^{+}$state to the $2590 \mathrm{keV}$ line shape. The best fit is obtained with a half-life of $T_{1 / 2}$ $=60.0 \pm 1.6$ (stat) \pm 6.0 (syst) fs for the $5958 \mathrm{keV}, 2_{2}^{+}$state. The line shape of the $5957 \mathrm{keV}$ peak was fit using this half-life and neutron branching ratios determined for the $2_{2}^{+} \rightarrow 2_{1}^{+}$transition together with the intensities of the $2_{2}^{+} \rightarrow 0_{1}^{+}$and $1^{-}$ $\rightarrow 0_{1}^{+}$transitions as free parameters. The best overall fit was obtained for intensities corresponding to branching ratios of $10 \pm 3 \%$ and $65 \pm 15 \%$, respectively in agreement with previously measured values $<10 \%$ and $83_{-6}^{+10} \%[10,11]$. The best fits (with $A_{2}=0$ ) for both transitions and the individual contributions of the three separate components to the line shapes, are shown in Fig. 3. At this point, we note that the $8.03 \mathrm{MeV}$ state in ${ }^{11} \mathrm{Be}$ is likely a $3 / 2^{-}$state as it feeds both the $0_{2}^{+}$and the $2_{2}^{+}$states, which could be reached by an $\ell$ $=1$ neutron. A $1 / 2^{-}$or $5 / 2^{-}$assignment is unlikely since it would require an $\ell=3$ neutron to reach one of the two states.

The effect of the $n-\gamma$ angular correlation was studied using the half-life and $A_{2}$ value as the only free parameters. The range of $A_{2}$ values corresponding to a mixed $E_{2} / M_{1}$ transition $\left(-0.68<A_{2}<0.68\right)$ was obtained from [19]. Assuming the worst case scenario that both neutron decay branches have angular correlations with the same sign of $A_{2}$, the minimum $\chi^{2} / \nu=0.9$ is obtained for $A_{2}=0 \pm 0.07$ corresponding to a half-life of $60 \pm 10 \mathrm{fs}$ (stat + syst). We note that although this range of $A_{2}$ values is consistent with that of a pure $E 2$ transition, this possibility can be ruled out because of the corresponding large $B\left(E 2 ; 2_{2}^{+} \rightarrow 2_{1}^{+}\right)$of about 57 Weisskopf units (W.u.).

The remainder of this paper focuses on the interpretation of the information obtained for the $2^{-}$and $2_{2}^{+}$states in ${ }^{10} \mathrm{Be}$ and discusses the nature of the $8.81 \mathrm{MeV}$ state in ${ }^{11} \mathrm{Be}$. The likely molecularlike structures of the four excited states in ${ }^{10} \mathrm{Be}\left(2_{2}^{+}, 1^{-}, 0_{2}^{+}, 2^{-}\right)$lying just below the ${ }^{9} \mathrm{Be}+n$ and ${ }^{6} \mathrm{He}+\alpha$ thresholds have recently been investigated in various theoretical studies [20-23]. The dominant structure of the two states $\left(2_{2}^{+}, 2^{-}\right)$fed by the neutron decay from the $8.81 \mathrm{MeV}$ state in ${ }^{11} \mathrm{Be}$ is found to be ${ }^{9} \mathrm{Be} \otimes 1 s_{1 / 2}$ for the $2^{-}$state and ${ }^{9} \mathrm{Be}$ $\otimes 0 p_{1 / 2}$ for the $2_{2}^{+}$state, i.e., a valence neutron, in a relative $s$ or $p$ wave, loosely bound to a ${ }^{9} \mathrm{Be}$ core, possibly in a halolike spatial distribution [21]. Recent work on the one-neutron knockout reaction $\left({ }^{11} \mathrm{Be},{ }^{10} \mathrm{Be}\right)$ has suggested that the $2^{-}$state is populated when a $p_{3 / 2}$ neutron is removed from the ${ }^{10} \mathrm{Be}$ core, leaving the $1 s_{1 / 2}$ valence (halo) neutron intact [24], suggesting that this state in ${ }^{10} \mathrm{Be}$ might be an excited state halo as proposed by [25]. Indeed, it shows remarkable similarity to the ground state of ${ }^{11} \mathrm{Be}$ with a single valence neutron in a $1 s_{1 / 2}$ state bound by about $0.5 \mathrm{MeV}$ to a ${ }^{9} \mathrm{Be}\left({ }^{10} \mathrm{Be}\right)$ core. Can we understand the structure of the $2_{2}^{+}$and $2^{-}$states in ${ }^{10} \mathrm{Be}$ in light of our $\beta$-decay study of ${ }^{11} \mathrm{Li}$ ?

It has been suggested [5] that if the $\beta$ decay of a halo nucleus takes place within its core then it is possible for the halo wave function to retain its features after the $\beta$ decay, even though the core may now have a rather different structure. The excitation energy of such a daughter state should then be roughly equal to the difference in $Q$ values of the decaying halo nucleus and its core. The $8.81 \mathrm{MeV}$ state in ${ }^{11} \mathrm{Be}$ is a strong candidate for just such a state and would thus have a large overlap with a ${ }^{9} \mathrm{Be}+n+n$ configuration. Support for this is provided from studies of the $\beta$-delayed twoneutron decay of ${ }^{11} \mathrm{Li}$, which is suggested to originate in part from this state [26,27]. In addition, this state is strongly populated in 2n-transfer reactions (see [28] and references therein). It is, however, clearly in contradiction to the interpretation $[20,28]$ that this state is part of a rotational band with a spin of $9 / 2^{-}$. The ${ }^{9} \mathrm{Be}$ core cannot be in its ground state, because this state lies $1.49 \mathrm{MeV}$ above the ${ }^{9} \mathrm{Be}+2 n$ threshold. The $8.81 \mathrm{MeV}$ state can however be built around a ${ }^{9} \mathrm{Be}$ excited state plus two neutrons coupled to spin 0 , namely the $5 / 2^{-}$state located $2.429 \mathrm{MeV}$ above the ground state of ${ }^{9} \mathrm{Be}$. The $Q_{\beta}$ of ${ }^{11} \mathrm{Li}-{ }^{11} \mathrm{Be}(8.81 \mathrm{MeV})$ and ${ }^{9} \mathrm{Li}$ ${ }^{9} \mathrm{Be}\left(5 / 2^{-}\right)$are only $635 \mathrm{keV}$ apart. A $5 / 2^{-}$spin assignment for the $8.81 \mathrm{MeV}$ state would explain why we do not observe any decay of the $8.81 \mathrm{MeV}$ state in ${ }^{11} \mathrm{Be}$ to the $1^{-}$state in ${ }^{10} \mathrm{Be}$, which is believed to have a similar structure to the $2^{-}$ state [21]. Instead, we observe its decay via single-neutron emission to just two bound excited states in ${ }^{10} \mathrm{Be}$ : the $2_{2}^{+}$and the $2^{-}$states with ${ }^{9} \mathrm{Be}+n$ separation energies of 854 and $548 \mathrm{keV}$, respectively. In each case, we suggest it is one of the original ${ }^{11} \mathrm{Li}$ halo neutrons $\left(55(10) \%\left(0 p_{1 / 2}\right)^{2}, 45(10) \%\right.$ $\left(1 s_{1 / 2}\right)^{2}$ [29]) that is emitted from the $8.81 \mathrm{MeV}$ state in ${ }^{11} \mathrm{Be}$, with the surviving valence neutron remaining in its $0 p_{1 / 2}$ or $1 s_{1 / 2}$ state and contributing to the structure of the $2_{2}^{+}$or $2^{-}$ states in ${ }^{10} \mathrm{Be}$, respectively. Indeed, shell model calculations by Brown [30] show that the $2_{2}^{+}$and $2^{-}$states have a strong 
$5 / 2^{-}$core component coupled with a $p_{1 / 2}$ and $s_{1 / 2}$ neutron, respectively. Note that we here neglect a possible (small) $\left(d_{5 / 2}\right)^{2}$ component in the ${ }^{11} \mathrm{Li}$ wave function, which could also populate the $2^{-}$state via $\ell=2$ neutron emission.

The possible halolike structure of the $2^{-}$state in ${ }^{10} \mathrm{Be}$ deserves special mention here as it is the best candidate to date of an excited neutron halo state. Our half-life measurement of $T_{1 / 2}=85 \pm 12 \mathrm{fs}$ corresponds to an experimental $B\left(E 1 ; 2^{-} \rightarrow 2_{1}^{+}\right)=7.7 \times 10^{-4}$ W.u. This value disagrees with the (preliminary) theoretical estimate of $0.02 \mathrm{~W} . \mathrm{u}$. obtained within a microscopic four cluster $(\alpha+\alpha+n+n)$ model using the resonating group method [31]. However, such a discrepancy is not surprising given the weakness of this transition. The theoretical value depends very sensitively on details of the wave functions of the two states involved; further theoretical work is in progress.

In summary, we have investigated the $\beta$ decay of ${ }^{11} \mathrm{Li}$ through the study of the Doppler-broadened transitions in ${ }^{10} \mathrm{Be}$ observed in the $\beta$-delayed one-neutron emission of ${ }^{11} \mathrm{Li}$. From the line-shape analysis of these transitions, we determined both the energies of the states in ${ }^{11} \mathrm{Be}$ feeding the excited states in ${ }^{10} \mathrm{Be}$ and also the half-life of some of these
${ }^{10} \mathrm{Be}$ excited states. We also find that the $\beta$ decay of ${ }^{11} \mathrm{Li}$ to the $8.81 \mathrm{MeV}$ state in ${ }^{11} \mathrm{Be}$ is likely to occur in the ${ }^{9} \mathrm{Li}$ core, leaving the two original halo neutrons of ${ }^{11} \mathrm{Li}$ unperturbed. The $8.81 \mathrm{MeV}$ state is interpreted as a ${ }^{9} \mathrm{Be}\left(5 / 2^{-}\right)$core plus the two surviving halo neutrons coupled to spin 0 . The oneneutron emission paths from the $8.81 \mathrm{MeV}$ state in ${ }^{11} \mathrm{Be}$ are consistent with the emission of one of the two halo neutrons and provide a supporting evidence that the $2_{2}^{+}$and $2^{-}$states in ${ }^{10} \mathrm{Be}$ have a halolike character, as predicted by various theoretical models.

This work was partially supported by the Natural Sciences and Engineering Research Council of Canada and the U.S. Department of Energy through Grant/Contract Nos. DEFG03-93ER40789 (Colorado School of Mines), DE-FG0296ER40958 (Georgia Tech) and W-7405-ENG-48 (University of California, Lawrence Livermore National Laboratory). We would like to thank the staff of the ISAC facility for their work in delivering the high-quality ${ }^{11} \mathrm{Li}$ beam. TRIUMF receives federal funding via a contribution agreement through the National Research Council of Canada.
[1] A. S. Jensen et al., Rev. Mod. Phys. 76, 215 (2004).

[2] P. G. Hansen and B. Jonson, Europhys. Lett. 4, 409 (1987).

[3] I. Tanihata et al., Phys. Rev. Lett. 55, 2676 (1985); Phys. Lett. 160B, 380 (1985).

[4] A. S. Jensen and K. Riisager, Phys. Lett. B 480, 39 (2000).

[5] N. K. Timofeyuk and P. Descouvemont, J. Phys. G 22, L99 (1996).

[6] D. J. Morrissey et al., Nucl. Phys. A627, 222 (1997).

[7] M. J. G. Borge et al., Phys. Rev. C 55, R8 (1997).

[8] N. Aoi et al., Nucl. Phys. A616, 181c (1997).

[9] H. O. U. Fynbo et al., Nucl. Phys. A736, 39 (2004).

[10] F. Ajzenberg-Selove, Nucl. Phys. A490, 1 (1988).

[11] D. Tilley et al., Energy Levels of Light Nuclei A=10 (2003). Available URL: http://www.tunl.duke.edu/nucldata/ourpubs/ p10_2003.pdf

[12] H. O. U. Fynbo, Nucl. Instrum. Methods Phys. Res. B 207, 275 (2003).

[13] G. Ball et al., Proceedings of the International Conference on the Nucleus: New Physics for the New Millenium, Faure, South Africa, edited by F. D. Smit, R. Lindsay, and S. V. Fortsch (Kluwer Academic/Plenum, New York, 1999) p. 69.

[14] C. E. Svensson et al., Nucl. Instrum. Methods Phys. Res. B 204, 660 (2003).
[15] D. C. Radford, Nucl. Instrum. Methods Phys. Res. A 361, 297 (1995).

[16] D. J. Millener et al., Phys. Rev. C 26, 1167 (1982).

[17] CERN, GEANT version 3.12 (1994).

[18] J. F. Ziegler and J. P. Biersack, SRIM-2003.10, http:// www.srim.org (2003).

[19] L. C. Biedenharn and M. E. Rose, Rev. Mod. Phys. 25, 729 (1953).

[20] W. vonOertzen, Z. Phys. A 357, 355 (1997).

[21] Y. Ogawa et al., Nucl. Phys. A673, 122 (2000).

[22] N. Itagaki et al., Phys. Rev. C 61, 044306 (2000); 65, 044302 (2002).

[23] Y. Kanada-En'yo and H. Horiuchi, Phys. Rev. C 66, 024305 (2002).

[24] T. Aumann et al., Phys. Rev. Lett. 84, 35 (2000).

[25] F. Negoita et al., Phys. Rev. C 59, 2082 (1999).

[26] R. Azuma et al., Phys. Rev. Lett. 43, 1652 (1979).

[27] F. M. Marques (private communication).

[28] H. G. Bohlen et al., Phys. At. Nucl. 65, 603 (2001).

[29] H. Simon et al., Phys. Rev. Lett. 83, 496 (1999).

[30] B. A. Brown (private communication).

[31] K. Arai (private communication). 